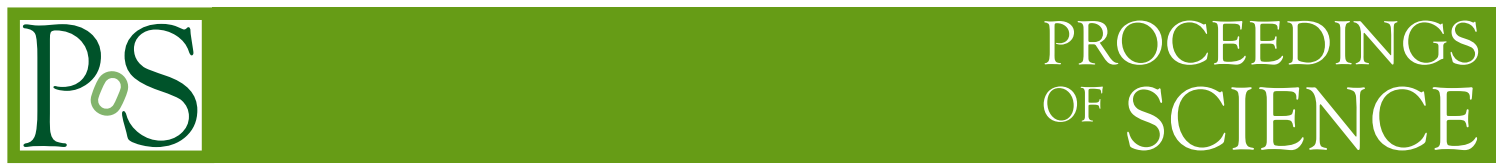

\title{
Quasi-Classical Model in SU(N) Gauge Field Theory
}

\section{A. Koshelkin*}

Moscow Institute for Physics and Engineering, Russia

E-mail: A Kosh@internets.ru

The quasi-classical model in a gauge theory with the Yang-Mills (YM) field is developed. On a basis of the exact solution of the Dirac equation in the $S U(N)$ gauge field, which is in the eikonal approximation, the Yang-Mills (YM) equations containing the external fermion current are solved. The derived solutions are quantized in the quasi-classical approach. The developed model proves to have the self-consistent solutions of the Dirac and Yang-Mills equations at $N \geq 3$. Thereat the solutions take place provided that the fermion and gauge fields exist simultaneously, so that the fermion current completely compensates the current generated by the gauge field due to it self-interaction. The obtained solution are considered in the context of QCD.

Light Cone 2010: Relativistic Hadronic and Particle Physics

June 14-18, 2010

Valencia, Spain

\footnotetext{
${ }^{*}$ Speaker.
} 


\section{Introduction}

The study of non-Abelian gauge fields plays an important role in the modern field theory[1, 2. 3]. The non-Abelian gauge field are a basis of QCD[4]. The knowledge of solutions of the YM equations enable us to understand specifics of processes in the strong interacting matter generated in collisions of heavy ions of high energies[5]. Primarily, this concerns studying the observable states of such matter as well as the processes accompanying evolution of the medium.

Studying the non-Abelian gauge fields has a very long history which was started by the classic paper by C.N.Yang and R.L.Mills[6]. Since the paper[6] has issued is a lot of papers[7, 8, 9, 10, 11, $12,13,14,15,16,17$ l have been devoted to deriving the solutions of the YM equation in various situations. The solution of the source-less YM equation in terms of plane waves was derived in [7, 8]. A wide class of solutions of the YM equation concerns $(1+3)$ Minkowski space-time in the presence of external sources[9, 10, 11, 12, 13]. In some specific case of $(1+2)$ space-time the YM equation were solved[14] for the $S U(2)$ gauge field. The spherical symmetric solutions are found for the $S U$ (2) fields in Ref.[15]. The Dirac equation in the presence of the $S U(3)$ YM field is considered[16, 17] in terms of studying the confinement problem. The quark confinement in the curve space-time is considered in Ref.[18]. Rather detailed review of the paper devoting to quantizing the YM field is in the monographs by A.Slavnov and L.Faddev[19].

Consistent consideration of strong interacting matter (generated, for example, in collisions of high energy ions) demands, generally, solving the Dirac and Yang-Mills equations simultaneously. The first step in studying such problem, naturally (see Ref.[20, 21]), is the attempt to derive the solution of such equations when the YM field has the form of some modified plane wave so that the both the Dirac and Yang-Mills fields will be in the confined region of space. The knowledge of the self-consistent solution of the Yang-Mills and Dirac equations in such approximation allows us to obtain the exact Green's function of a fermion field. As a result, it enables to drive both the renormalized vertex functions and effective mass of a fermion as well as to calculate the observable characteristics of the strong interacting matter generated in collisions of high energy ions [5] beyond the perturbation theory.

In the present paper the quasi-classical model in the $S U(N)$ gauge theory with the Yang Mills field is developed. The self-consistent solution of both the nonhomogeneous Yang-Mills equation and Dirac equations in an external field are derived when the gauge Yang-Mills field is in the eikonal form. It is shown that the self-consistent solutions of such equations takes place when $N \geq 3$. They occur provided that the fermion and gauge fields exist simultaneously, so that the fermion current completely compensates the current generated by the gauge field due to its self-interaction. Thereat there is no energy flux from the range of space where the fields are localized. Thereat, interaction between the fermions and YM field leads, in the mean, to the re-normalization a fermion mass. The re-normalized mass depends strongly on the temperature of matter.

\section{The YM equations in the presence of external current}

We consider the $S U(N)$ gauge field $A_{a}^{v}$ generated by a fermion current. It satisfies the following equations[19, 22]: 


$$
\begin{aligned}
& \partial_{\mu} F_{a}^{v \mu}(x)-g \cdot f_{a b}^{c} A_{\mu}^{b}(x) F_{c}^{v \mu}(x)=-g J_{a}{ }^{v}(x) \\
& F_{a}^{v \mu}(x)=\partial^{v} A_{a}^{\mu}(x)-\partial^{\mu} A_{a}^{v}(x)-g \cdot f_{a}^{b c} A_{b}^{v}(x) A_{c}^{\mu}(x), \\
& J_{a}{ }^{v}(x)=\bar{\Psi}(x) \gamma^{v} T_{a} \Psi(x),
\end{aligned}
$$

where the fermion fields $\Psi(x), \bar{\Psi}(x)$ are governed by the Dirac equation:

$$
\begin{aligned}
& \left\{i \gamma^{\mu}\left(\partial_{\mu}+i g \cdot A_{\mu}^{a}(x) T_{a}\right)-m\right\} \Psi(x)=0 \\
& \bar{\Psi}(x)\left\{i \gamma^{\mu}\left(\overleftarrow{\partial}_{\mu}-i g \cdot A_{\mu}^{* a}(x) T_{a}\right)+m\right\}=0 ; \quad T_{a}=\frac{1}{2} \lambda_{a} .
\end{aligned}
$$

Here, $m$ is a fermion mass, $g$ is the coupling constant; $\gamma^{v}$ are the Dirac matrixes [23, 24], $x \equiv$ $x^{\mu}=\left(x^{0} ; \vec{x}\right)$ is a vector in the Minkowski space-time; $\partial_{\mu}=(\partial / \partial t ; \nabla)$; the Roman letters numerate a basis in the space of the associated representation of the $S U(N)$ group, so that $a, b, c=1 \ldots N^{2}-$ 1. We use the signature $\operatorname{diag}\left(\mathscr{G}^{\mu \nu}\right)=(1 ;-1 ;-1 ;-1)$ for the metric tensor $\mathscr{G}^{\mu \nu}$. The line and "dagger" over $\Psi$ mean the Dirac and hermitian conjugation, respectively[17]. Summing over any pair of the repeated indexes is implied.

The symbols $T_{a}$ in Eqs.(2.3)-(2.5) are the generators of the $S U(N)$ group which satisfy the commutative relations and normalization condition:

$$
\begin{aligned}
& {\left[T_{a}, T_{b}\right]_{-}=T_{a} T_{b}-T_{b} T_{a}=i f_{a b}{ }^{c} T_{c} ; \quad f_{a b}{ }^{c}=-2 i \operatorname{Tr}\left(\left[T_{a}, T_{b}\right]_{-} T_{c}\right)} \\
& \operatorname{Tr}\left(T_{a} T_{b}\right)=\frac{1}{2} \delta_{a b}
\end{aligned}
$$

where $f_{a b}{ }^{c}$ are the structure constant of the $S U(N)$ group, which are real and anti-symmetrical with respect to the transposition in any pair of indexes; $\delta_{a b}$ is the Kroneker symbol. In the matrix representation the operators $\left(2 T_{a}\right)$ coincide with the Pauli and Gill-Mann matrixes when $N$ is equal to 2 or 3 , respectively.

It directly follows from Eqs.(2.6), (2.7) that

$$
\left[T_{a}, T_{b}\right]_{+}=T_{a} T_{b}+T_{b} T_{a}=\frac{1}{N} \delta_{a b}+d_{a b c} T^{c}, \quad d_{a b c}=2 \operatorname{Tr}\left(\left[T_{a}, T_{b}\right]_{+} T_{c}\right)
$$

where $d_{a b c}$ is real and symmetrical with respect to the transposition in any pair of indexes.

The main goal is to derive the self-consistent solutions of of Eqs.(2.1)-(2.5) which will be localized in the confined region of space. We find the solution when the field $A_{v}^{a}(x)$ is in the form:

$$
A_{a}^{v}(x)=A_{a}^{v}(\varphi(x))
$$


where $\varphi(x)$ is some scalar function in the Minkowski space-time which is such that:

$$
\left(\partial_{\mu} \varphi\right)\left(\partial^{\mu} \varphi\right) \equiv k_{\mu} k^{\mu}=0
$$

The last formula determines the well known eikonal approximation where $\varphi(x)$ can be interpretable as the function governing the wave surface of the field $A_{a}^{v}$.

We take the axial gauge for the field $A_{\mu}^{a}(x)$ :

$$
\partial^{\mu} A_{\mu}^{a}=0 ; \quad k^{\mu} \dot{A}_{\mu}^{a}=0
$$

where the dot over the letter means differentiation with respect to the introduced variable $\varphi$.

Taking into account of both the dependence of $A_{v}^{a}(x)$ on the variable $x$ via the function $\varphi(x)$ and formulae (2.10), (2.11), we derive from Eqs.(1), (2):

$$
\begin{aligned}
& -\left(\partial_{\mu} \partial^{\mu} \varphi(x)\right) \cdot \dot{A}_{a}^{v}-g k^{v} f_{a b}{ }^{c} A_{\mu}^{b}(\varphi) \dot{A}_{c}^{\mu}(\varphi)+g^{2} f_{a b}^{c} f_{c}{ }^{s r}\left\{A_{\mu}^{b}(\varphi) A_{s}^{v}(\varphi) A_{r}^{\mu}(\varphi)\right\}=-g J_{a}^{v}(x) \\
& J_{a}^{v}(x)=\bar{\Psi}(x) \gamma^{v} T_{a} \Psi(x) .
\end{aligned}
$$

It follows from Eq.(2.12) that in order to derive the solution of the YM equation it is necessary to calculate the fermion current $J_{a}^{v}(x)$, given by Eq.(2.3). We assume that the field $A_{a}^{v}(\varphi)$ can expanded as follows in the local frame:

$$
\begin{aligned}
& A_{a}^{v}(\varphi)=A\left(e_{(1)}^{v}(\varphi) \cos \left(\varphi(x)+\varphi_{a}\right)+e_{(2)}^{v}(\varphi) \sin \left(\varphi(x)+\varphi_{a}\right)\right)+\mathscr{B}_{a} \partial^{v} \varphi(x) \\
& e_{(1)}^{v} k_{v}=e_{(2)}^{v} k_{v}=0 ; \quad \dot{e}_{(1)}^{v}=e_{(2)}^{v} ; \quad \dot{e}_{(2)}^{v}=-e_{(1)}^{v} \quad ; k^{v} \equiv \partial^{v} \varphi(x),
\end{aligned}
$$

where $e_{(1),(2)}^{v}(\varphi)$ are the space-like 4-vectors on the wave surface $\varphi(x)$ which are independent on the group variable $a$; the symbols $A, \mathscr{B}_{a}$ and $\varphi_{a}$ are the constants in the Minkowski space-time. They are determined via the initial condition of the studied problem. It is obvious that the function $\varphi(x)$ can be taken so that the field $A_{v}^{a}(x)$ will be localized in the confined region of space.

\section{Fermions in the external YM field}

To obtain the fermion field $\Psi(x)$ we go from Eq.(2.4) to the so-called quadric Dirac equation which has the following form:

$$
\begin{aligned}
& \left\{-\partial_{\mu} \partial^{\mu}-m^{2}+g^{2}\left(\gamma^{\mu} A_{\mu}^{a} T_{a}\right)^{2}+2 i g\left(\gamma^{\mu} A_{\mu}^{a} T_{a}\right)\left(\gamma^{\mu} \partial_{\mu}\right)+i g\left(\gamma^{\mu} k_{\mu}\right)\left(\gamma^{\mu} \dot{A}_{\mu}^{a} T_{a}\right)\right\} \Phi(x)=0 ; \\
& \Psi(x)=\left\{\frac{i \gamma^{\mu}\left(\partial_{\mu}-i g \cdot A_{\mu}^{a}(x) T_{a}\right)+m}{2 m}\right\} \Phi(x)
\end{aligned}
$$

First, to derive the solution of the last equation we simplify the third term in the left-hand side of Eq.(3.1). 
Let the initial conditions be so that the phases $\varphi_{a}$ in Eq.(2.13) satisfy the equations:

$$
d_{a}^{b c} \cos \left(\varphi_{a}-\varphi_{b}\right)=0 .
$$

The last formula means that different generators $T_{a}$ rotate spinors independently in spite of interaction of a fermion with an external field.

Then, using Eqs.(2.6)-(2.8) and relations for the $\gamma$-matrixes [23, 24] we obtain after the direct calculations:

$$
\begin{aligned}
& \left(\gamma^{\mu} A_{\mu}^{a} T_{a}\right)^{2}=\left(\frac{1}{2 N} \delta_{a b}+\frac{1}{2} d_{a b c} T^{c}+\frac{i}{2} f_{a b c} T^{c}\right)\left(\mathscr{G}^{\mu v}+\sigma^{\mu v}\right) A_{\mu}^{a} A_{v}^{b}=\frac{1}{2 N} A_{\mu}^{a} A_{a}^{\mu} ; \\
& \sigma^{\mu v}=\frac{1}{2}\left(\gamma^{\mu} \gamma^{v}-\gamma^{v} \gamma^{\mu}\right)
\end{aligned}
$$

We note, that the term containing $\left(f_{a b c} T^{c} \sigma^{\mu v} A_{\mu}^{a} A_{v}^{b}\right)$ disappears since the vectors $e_{(1),(2)}^{v}$ in the expansion (13) are independent on the group variable $a$. The same takes place in Eq.(3.1) when the term contains $\sigma^{\mu \nu}$.

Let us find the solution of Eq.(3.1) in the following form:

$$
\Phi(x) \equiv \Phi_{\sigma, \alpha}(x, p)=e^{-i p x} \cdot F_{\sigma, \alpha}(\varphi) .
$$

where $F_{\sigma, \alpha}(\varphi)$ is some multicomponent function which is the generalized Dirac spinor. It depends on both the spin variable $\sigma$ and the variable $\alpha$ which specifies the state of a fermion in the space of the fundamental representation of the $S U(N)$ group, thereat $\alpha=1 \div N ; p^{v}=\left(p^{0}, \vec{p}\right)$ is some 4-vector.

We substitute $\Phi_{a, \alpha}(x, p)$ as Eq.(3.4) into the formula (3.1). Using the relations for the $\gamma$ matrixes [23, 24], the independence of $e_{(1),(2)}^{v}$ on the group variable $a$ in the local frame (see Eq.(2.13)) as well as Eq.(2.10), (2.11), (3.3), we obtain:

$$
\begin{aligned}
& \left\{p^{2}-m^{2}-\frac{g^{2}\left(N^{2}-1\right) A^{2}}{2 N}-2 g\left(T_{a} A_{\mu}^{a} p^{\mu}\right)-i g\left(\gamma^{\mu} k_{\mu}\right)\left(\gamma^{\mu} T_{a} \dot{A}_{\mu}^{a}\right)\right\} F_{\sigma, \alpha}(\varphi)+i(p k) \dot{F}_{\sigma, \alpha}(\varphi)=0 ; \\
& k_{\mu}=\partial_{\mu} \varphi(x) ; \quad(p k)=p^{\mu} k_{\mu} ;
\end{aligned}
$$

where the dot over $\dot{F}_{\sigma, \alpha}(\varphi)$ means the derivative with respect to the variable $\varphi$.

In obtaining the last equation we neglect $\left|\partial_{\mu} k^{\mu}\right|$ as compared with $|(p k)|$ (see Eq.(3.5)). This means that the wave length $\lambda_{Y M}$ of the YM field is unchangeable on the scale which is of the order of the de Broglie wave length of a fermion $\lambda_{F}$ :

$$
\left|\partial_{\mu} k^{\mu}\right|=\left|\partial_{\mu} \partial^{\mu} \varphi(x)\right| \sim\left|\frac{d \lambda_{Y M}}{\lambda_{Y M}^{2} d x}\right| \ll \frac{1}{\lambda_{Y M} \lambda_{F}} \sim|(p k)| \Leftrightarrow\left|\frac{d \lambda_{Y M}}{d x}\right| \ll \frac{\lambda_{Y M}}{\lambda_{F}} \ll 1
$$

The last inequality corresponds to the so called quasi-classical approximation and means that $\partial_{\mu} k^{\mu}=0$. The condition $\partial_{\mu} k^{\mu}=0$ can be treated as the scale invariance of the wave surface of 
the YM field. Thereat, the form of the wave surface is determined by some harmonic functions satisfying D'Lambert equation $\partial_{\mu} \partial^{\mu} \varphi(x)=0$.

The solution of Eq.(3.5) can be written in the form:

$$
\begin{aligned}
& F_{\sigma, \alpha}(\varphi)=\exp \left(-i g^{2} \frac{\left(N^{2}-1\right) A^{2}}{2 N(p k)} \varphi\right) \exp \left\{-i g T_{a} \frac{\int_{0}^{\varphi} d \varphi^{\prime}\left(A_{\mu}^{a} p^{\mu}\right)+\frac{i}{2}\left(\gamma^{\mu} k_{\mu}\right)\left(\gamma^{\mu} A_{\mu}^{a}\right)}{(p k)}\right\} u_{\sigma}(p) \cdot v_{\alpha}, \\
& p^{2}=m^{2} ; \quad \partial_{\mu} k^{\mu}=\partial_{\mu} \partial^{\mu} \varphi(x)=0 ;
\end{aligned}
$$

where $u_{\sigma}(p)$ and $v_{\alpha}$ are some spinors which are the elements of spaces of the corresponding representations.

The second exponent in Eq.(3.7) is an operator acting on the spinors $u_{\sigma}(p)$ and $v_{\alpha}$. The transformation of it is absolutely analogous to the calculations made in Ref.[25] and consists of separating the groups of odd and even terms in the expansion of the exponent (see Ref.[25]). As a result, we obtain:

$$
\begin{aligned}
& \Psi_{\sigma, \alpha}(x, p)= \\
& \Phi_{\sigma, \alpha}(x, p)=\cos \theta \cdot \exp \left(-i g^{2} \frac{\left(N^{2}-1\right) A^{2}}{2 N(p k)} \varphi-i p x\right)\left\{\left(1-i g T_{a} \frac{\tan \theta}{\theta(p k)} \int_{0}^{\varphi} d \varphi^{\prime}\left(A_{\mu}^{a} p^{\mu}\right)\right)+\frac{g\left(\gamma^{\mu} k_{\mu}\right) \overline{\left(\gamma^{\mu} A_{\mu}^{a}\right)}}{2(p k)} .\right. \\
& \left.\left[\frac{\tan \theta}{\theta} T_{a}+\frac{g}{(p k)} \frac{1}{2 N} \int_{0}^{\varphi} d \varphi^{\prime}\left(A_{\mu}^{a} p^{\mu}\right)\left(-i \frac{\tan \theta}{\theta}+\frac{g}{(p k)} \frac{\theta-\tan \theta}{\theta^{3}} T_{b} \int_{0}^{\varphi} d \varphi^{\prime}\left(A_{\mu}^{b} p^{\mu}\right)\right)\right]\right\} u_{\sigma}(p) \cdot v_{\alpha} .
\end{aligned}
$$

Following the structure of the last formula it is naturally to determine the spinor $u_{\sigma}(p)$ as the standard Dirac spinors which satisfy the free Dirac equation and are normalized as follows:

$$
\bar{u}_{\sigma}(p) u_{\lambda}\left(p^{\prime}\right)= \pm 2 m \delta_{\sigma \lambda} \delta_{p p^{\prime}} ; \quad p^{2}=m^{2}
$$

where the plus and minus signs correspond to the Dirac scalar production of the spinors $u_{\sigma}(p)$ and $u_{\sigma}(-p)$, respectively.

As for the spinor $v_{\alpha}$ we determine it by the relations:

$$
v_{\alpha}^{\dagger} v_{\beta}=\delta_{\alpha \beta} ; \quad \operatorname{Tr}\left(T_{a}\right)=0 ; \quad \operatorname{Tr}\left(T_{a} T_{b}\right)=\frac{1}{2} \delta_{a b}
$$

The function (3.8) can be normalized by the $\delta$-function as follows:

$$
\int d^{3} x \Psi_{\sigma, \alpha}^{*}\left(x, p^{\prime}\right) \Psi_{\sigma, \alpha}(x, p)=(2 \pi)^{3} \delta^{3}\left(\vec{p}-\vec{p}^{\prime}\right)
$$


They allow us to drive the mean value $Q^{\mu}$ of the kinetic momentum of a fermion $p^{\mu}-g T^{a} A_{a}^{\mu}$. After a direct calculations we get

$$
\left(Q^{0}\right)^{2}=\vec{p}^{2}+m_{*}^{2} ; \quad m_{*}^{2}=m^{2}+\frac{g^{2}\left(N^{2}-1\right) A^{2}}{2 N}
$$

Interpreting $Q^{0}$ as the mean value of the energy $E$ of a fermion in an external field, we conclude from the last equation that the interaction of a fermion with an external field leads, in the mean, to the re-normalization of a fermion mass.

The direct calculation shows that $\Phi_{\sigma, \alpha}(x, p)$ and $\Phi_{-\sigma, \alpha}(x,-p)$ are orthogonal. In this way, it is obvious, that $\Phi_{\sigma, \alpha}(x, p)$ is the so-called positively frequency function whereas $\Phi_{-\sigma, \alpha}(x,-p)$ is negatively frequency one[16, 17]. This fact allows us to construct the general solution of the Dirac equation which describes the states both particles and anti-particles. As a result, the general solution of Eq.(3.1) provided that $\partial_{\mu} k^{\mu}=0$ is

$$
\begin{aligned}
& \Psi(x)=\sum_{\sigma, \alpha} \int \frac{d^{3} p}{\sqrt{2 p^{0}}(2 \pi)^{3}}\left\{\hat{a}_{\sigma, \alpha}(\vec{p}) \Psi_{\sigma, \alpha}(x, p)+\hat{b}_{\sigma, \alpha}^{\dagger}(\vec{p}) \Psi_{-\sigma, \alpha}(x,-p)\right\} \\
& \bar{\Psi}(x)=\sum_{\sigma, \alpha} \int \frac{d^{3} p}{\sqrt{2 p^{0}}(2 \pi)^{3}}\left\{\hat{a}_{\sigma, n \alpha}^{\dagger}(\vec{p}) \bar{\Psi}_{\sigma, \alpha}(x, p)+\hat{b}_{\sigma, \alpha}(\vec{p}) \bar{\Psi}_{-\sigma, \alpha}(x,-p)\right\}
\end{aligned}
$$

where the symbols $\hat{a}_{\sigma, \alpha}^{\dagger}(\vec{p}) ; \hat{b}_{\sigma, \alpha}^{\dagger}(\vec{p})$ and $\hat{a}_{\sigma, \alpha}(\vec{p}) ; \hat{b}_{\sigma, \alpha}(\vec{p})$ are the operators of creation and cancellation of a fermion $\left(\hat{a}_{\sigma, \alpha}(\vec{p}) ; \hat{a}_{\sigma, \alpha}^{\dagger}(\vec{p})\right)$ and anti-fermion $\left(\hat{b}_{\sigma, \alpha}(\vec{p}) ; \hat{b}_{\sigma, \alpha}^{\dagger}(\vec{p})\right)$, respectively[22, 23]. Thereat, $\hat{a}_{\sigma, \alpha}(\vec{p}) \hat{I}^{1} \hat{a}_{\sigma, \alpha}^{\dagger}(\vec{p}) ; \hat{b}_{\sigma, \alpha}(\vec{p})$ and $\hat{b}_{\sigma, \alpha}^{\dagger}(\vec{p})$ satisfy the standard commutative relations for fermion operators.

\section{Solution of YM equation in the eikonal approximation}

Let us fix the state of fermion vacuum so that the bilinear combinations of the operators of creation and cancellation of fermions are diagonal. Substituting $\bar{\Psi}(x)$ and $\Psi(x)$ given by Eq.(3.8), (3.13) into the formula (2.3) we derive the fermion current $J_{a}^{v}$. When the fermion system is homogeneous and isotropic the current is proportional to the vector $A_{a}^{v}$. Taking into account of the explicit form of the field $A_{a}^{v}$ given by Eq.(2.13), we substitute the derived current $J_{a}^{v}$ into Eq.(2.12). As a result, we obtain:

$$
\begin{gathered}
2 f_{a b}{ }^{c} \sin \left(\varphi_{b}-\varphi_{c}\right)=f_{a b}{ }^{c}\left\{f_{c}{ }^{s r} \cos \left(\varphi_{b}-\varphi_{r}\right)+\left\{\cos \left(\varphi_{b}-\varphi_{r}\right) \cos \left(\varphi_{s}-\varphi_{a}\right)\right\} \frac{f_{c}{ }^{b s}}{N}\right\} \mathscr{B}_{s} \\
A^{2} \cdot C=-\left(N^{2}-1\right) \sum_{\sigma \alpha} \int \frac{d^{3} p}{p^{(0)}(2 \pi)^{3}}\left\langle\hat{a}_{\sigma, \alpha}^{\dagger}(\vec{p}) \hat{a}_{\sigma, \alpha}(\vec{p})+\hat{b}_{\sigma, \alpha}(\vec{p}) \hat{b}_{\sigma, \alpha}^{\dagger}(\vec{p})\right\rangle,
\end{gathered}
$$

where 


$$
C=f_{a b}^{c} f_{c}^{s r}\left\{\cos \left(\varphi_{b}-\varphi_{r}\right) \cos \left(\varphi_{s}-\varphi_{a}\right)\right\}<0 .
$$

The equations (4.1), (4.2) are closed with respect to the unknown quantities $A$ and $\mathscr{B}_{a}$. Having been solved they determine both the fermion and gauge field by means of Eqs.(2.13), (3.8), (3.13) so that the wave surface $\varphi(x)$ is governed by relations (2.10), (3.6), (3.7).

Note that in the case of the $N=2$ (when the $S U(2)$ gauge symmetry occurs) the convolution (4.3) containing cosines is always positive since the structure constants $f_{a b}{ }^{c}$ are the completely antisymmetrical tensor of the third rang $\varepsilon_{a b}^{c}$ due to the Jacob equality[22]:

$$
C=f_{a b}^{c} f_{c}^{s r}\left\{\cos \left(\varphi_{b}-\varphi_{r}\right) \cos \left(\varphi_{s}-\varphi_{a}\right)\right\}=\sum_{a, b=0}^{N^{2}-1} \sin ^{2}\left(\varphi_{s}-\varphi_{a}\right) \geq 0 .
$$

This means that in the framework of the developed model there is no self-consistent solution of the Dirac and Yang-Mills equations for the $S U(2)$ gauge symmetry. In the cases of the groups whose dimension is more then $N=2$ the structure constants $f_{a b}{ }^{c}$ can not be expressed in terms of the tensor $\varepsilon_{a b}{ }^{c}$. As a result, it possible to fix the differences between phase in the convolution $C$ so that $C \leq 0$.

As for the coefficients $\mathscr{B}_{s}$ they satisfy the set of linear algebraical equations. The matrix of this set is symmetrical and, moreover, its diagonal elements are not all equal to zero. This means that the equation for $\mathscr{B}_{s}$ has the only solution.

As a result, we have the following. The problem governed by Eqs.(2.1)-(2.5) has the only solution when $N \geq 3$. The solutions are determined by Eqs.(2.13), (3.13), (4.1), (4.2) and correspond to the eikonal consideration when the wave surface of the fields are determined by the equations:

$$
\left(\partial_{\mu} \varphi(x)\right) \cdot\left(\partial^{\mu} \varphi(x)\right)=0 ; \quad\left(\partial_{\mu} \partial^{\mu}\right) \varphi(x)=0
$$

It follows from Eqs. (2.13), (3.13), (4.1), (4.2) that the Yang-Mills and Dirac equations has the self-consistent solution when the fermion current compensates the current of the gauge field which appears due to self-interaction of such field. In other words, in the the framework of the developed model there is no the YM field without fermions. In terms of QCD this means that quarks and gluons can not exist separately in such approach.

We should note here that the second relation in Eq.(4.5) implies that the function $\varphi(x)$ which is the argument in the expansion (2.13) of the field $A_{a}^{v}$ is the so called harmonic function. Owing to the initial conditions it can be always taken such that the field $A_{a}^{v}$ will be localized in the confined region of space.

\section{Developed model in context of QCD}

First, we discuss the applicability of the developed model to description of the strong interacting matter generated in collisions of heavy ions of high energies. The quasi-classicality of the model means that the occupancy number of particle are large. 
In the RHIC and SPS experiments the characteristic temperature $T$ of an equilibrium quarkgluon plasma is $T \sim 200 \div 400 \mathrm{MeV}$. The estimations of the initial density of energy of the plasma give that the energy density $w \sim 10 \mathrm{Gev} \cdot \mathrm{F}^{-3}$ while the volume of a fireball is not less than $V_{0} \sim$ $10^{2} F^{3}$. Then the number of particles $N_{0}$ inside the fireball is of the order of

$$
N_{0} \sim \frac{w V_{0}}{T} \gtrsim 2.5 \cdot 10^{3}
$$

that is in agreement with the quasi-classical approximation.

The gas parameter $n_{0}^{1 / 3} T^{-1}$ is of the order of $\left(n_{0}^{1 / 3} T^{-1}\right) \sim 1.46 \div 3.7$ at such density of the matter. On the other hand, the mean effective mass of a quark is of the order of

$$
\begin{aligned}
m_{*} & \sim\left(\frac{2 N}{g\left(N^{2}-1\right)}\right)^{\frac{1}{2}}\left(\frac{n_{0}}{2|C| T^{3}}\right)^{1 / 2} T ; \quad \frac{m}{T} \ll\left(\frac{n_{0}}{|C| T^{3}}\right) \ll 1 ; \\
m_{*} & \sim \sqrt[3]{n_{0}} ; \quad\left(\frac{n_{0}}{|C| T^{3}}\right) \gg 1 .
\end{aligned}
$$

It follows from the last formulae that in the intermediate range of the density of matter $n_{0} \sim$ $(g T)^{3}$ the effective mass is proportional to the temperature of the matter that corresponds to the result of the calculation of the thermal mass of a quark in the hard loop approximation[26, 27]:

$$
m_{*} \sim g T
$$

\section{Conclusion}

The quasi-classical model in the gauge $S U(N)$ field theory is considered when the YM field is assumed to be in form of the eikonal wave. The self-consistent solutions of the non-homogeneous YM equation and the Dirac equation in the external YM field is derived.

It is shown that the considered problem is solvable when the dimension of the gauge group $N \geq 3$. Thereat, the currents generated by fermions and gauge field exactly compensate each other.

We shown that the developed model corresponds to the quasi-classical consideration of the problem. In the case of an equilibrium matter which consists of quarks and gluons, such approximation corresponds to the smallness of wave length of the YM field as compared with the inverse temperature of the matter.

The relation of the developed model to the generally accepted point of view on the matter generated in collisions of heavy ions of high energies is considered. In the case of the hot homogeneous equilibrium quark-gluon plasma the re-normalization of a fermion mass leads to appearing the thermal mass of a quark which strongly depends on the matter temperature. We show that in the intermediate range of the density and temperature of the plasma, $n_{0} \sim g^{3} T^{3}$, the dependence of the quark mass on the matter temperature and coupling constant (see Eq.(5.3)) corresponds to the results of the calculation of it which have been made in the hard thermal loop approximation[26, 27] early. 


\section{References}

[1] P.H.Frampton, Gauge Field Theories, Second Edition, Wiley, 2000.

[2] D.Gross, Chineses J. of Physics, 30, 955 (1992).

[3] A.M.Polyakov, Gauge Field and Strings. Harwood Academic Publishers, Chur, Switzerland, 1987

[4] F.J.Yndurain. The Theory of Quarks and Gluons Interaction, Springer, 2006.

[5] D.E.Kharzeev, Nucl.Phys. A 69995 (2002); D.E.Kharzeev, J.Raufeisen, nucl-th/0206073, v1, 28 June, 2002.

[6] C.N.Yang, R.L.Mills, Rev. 51461 (1954).

[7] S.Coleman. Phys. Lett.,B 70, 59 (1977).

[8] T.Eguchi. Phys. Rev.,D 13, 1561 (1976).

[9] P.Sikivie, N.Weiss, Phys. Rev. 20487 (1979).

[10] R.Jackiw, L.Jacobs, C.Rebbi, Phys. Rev. D21 426 (1980 ).

[11] R.Teh, W.K.Koo, C.H.Oh, Phys. Rev. D24 2305 (1981) ; Phys. Rev. D25 3263 (1982).

[12] D.Sivers, Phys. Rev. D34 1141 (1986).

[13] V.M.Vyas,T.S.Raju, T.Shreecharan. e-Print: arXiv:0912.3993 [het-th].

[14] C.H. Oh, L.H. Sia, R.Teh, Phys. Rev. D40 601 (1989).

[15] R.Bartnik, J.McKinnon, Phys. Rev. Lett. 61141 (1988).

[16] Y.Brihaye, E.Radu, D.H.Tchrahian, Phys. Rev. D75 024022 (2007).

[17] D.D.Dietrich, Phys Rev. D 80 (2009) 067701.

[18] C.C.Barros Jr., Eur. Phys. J., C42 1196 (2005).

[19] A.Slavnov, L.Faddeev, Introduction to Quantum Theory of Gauage Fields, 2nd enl. and rev. ed. Moscow, Nauka, 1988.

[20] E.S.Fradkin, Nucl.Phys. 76 (1966) 588.

[21] O.Oron, L.P.Horwitz, Gen. Relativ. Gravit. 37(3) (2005) 491506.

[22] A.I.Akhiezer, S.V.Peletminsky. The field and Fundanmetal Interactions. Kiev, Naukova Dumka, 1986.

[23] V.B.Berestetzkii, E.M.Lifshitz, L.P.Pitaevskii. Quantum Electrodynamic, Pergamon Press, 1981.

[24] C.Itzykson, J.-B.Zuber. Quantum Field Theory. McGrow-Hill Book Company, 1980.

[25] A.V.Koshelkin. Phys. Lett. B 683 (2010) 205. 1974.

[26] E.Braaten, R.D.Pisarski, Mucl. Phys. B337 596 (1990).

[27] J.Frenkel, J.C.Taylor, Nucl. Pys. B 334199 (1990). 